\title{
Evidence for a chemically differentiated outflow in Mrk $231^{\star, \star \star}$
}

\author{
J. E. Lindberg ${ }^{1,2, \star \star \star}$, S. Aalto ${ }^{1}$, S. Muller ${ }^{1}$, I. Martí-Vidal ${ }^{1}$, N. Falstad ${ }^{1}$, F. Costagliola ${ }^{1,3}$, C. Henkel $^{4,5}$, \\ P. van der Werf ${ }^{6}$, S. García-Burillo ${ }^{7}$, and E. González-Alfonso ${ }^{8}$
}

\author{
${ }^{1}$ Department of Earth and Space Sciences, Chalmers University of Technology, Onsala Observatory, 43994 Onsala, Sweden \\ e-mail: johan. lindberg@nasa.gov \\ 2 NASA Goddard Space Flight Center, Astrochemistry Laboratory, Mail Code 691, 8800 Greenbelt Road, Greenbelt, \\ MD 20771, USA \\ 3 Osservatorio di Radioastronomia (ORA-INAF), Italian ALMA Regional Centre, c/o CNR, via Gobetti 101, 40129 Bologna, Italy \\ ${ }^{4}$ Max-Planck-Institut für Radioastronomie, Auf dem Hügel 69, 53121 Bonn, Germany \\ 5 Astronomy Department, King Abdulaziz University, PO Box 80203, 21589 Jeddah, Saudi Arabia \\ ${ }^{6}$ Leiden Observatory, Leiden University, PO Box 9513, 2300 RA Leiden, The Netherlands \\ 7 Observatorio Astronómico Nacional (OAN-IGN)-Observatorio de Madrid, Alfonso XII, 3, 28014 Madrid, Spain \\ 8 Universidad de Alcalá de Henares, Departamento de Física, Campus Universitario, 28871 Alcalá de Henares, Madrid, Spain
}

Received 27 September 2015 / Accepted 14 December 2015

\section{ABSTRACT}

\begin{abstract}
Aims. Our goal is to study the chemical composition of the outflows of active galactic nuclei and starburst galaxies.
Methods. We obtained high-resolution interferometric observations of $\mathrm{HCN}$ and $\mathrm{HCO}^{+} J=1 \rightarrow 0$ and $J=2 \rightarrow 1$ of the ultraluminous infrared galaxy Mrk 231 with the IRAM Plateau de Bure Interferometer. We also use previously published observations of $\mathrm{HCN}$ and $\mathrm{HCO}^{+} J=1 \rightarrow 0$ and $J=3 \rightarrow 2$, and $\mathrm{HNC} J=1 \rightarrow 0$ in the same source.

Results. In the line wings of the $\mathrm{HCN}, \mathrm{HCO}^{+}$, and $\mathrm{HNC}$ emission, we find that these three molecular species exhibit features at distinct velocities which differ between the species. The features are not consistent with emission lines of other molecular species. Through radiative transfer modelling of the $\mathrm{HCN}$ and $\mathrm{HCO}^{+}$outflow emission we find an average abundance ratio $X(\mathrm{HCN}) / X\left(\mathrm{HCO}^{+}\right) \gtrsim 1000$. Assuming a clumpy outflow, modelling of the $\mathrm{HCN}$ and $\mathrm{HCO}^{+}$emission produces strongly inconsistent outflow masses.

Conclusions. Both the anti-correlated outflow features of $\mathrm{HCN}$ and $\mathrm{HCO}^{+}$and the different outflow masses calculated from the radiative transfer models of the $\mathrm{HCN}_{\text {and }} \mathrm{HCO}^{+}$emission suggest that the outflow is chemically differentiated. The separation between $\mathrm{HCN}$ and $\mathrm{HCO}^{+}$could be an indicator of shock fronts present in the outflow, since the $\mathrm{HCN} / \mathrm{HCO}^{+}$ratio is expected to be elevated in shocked regions. Our result shows that studies of the chemistry in large-scale galactic outflows can be used to better understand the physical properties of these outflows and their effects on the interstellar medium in the galaxy.
\end{abstract}

Key words. galaxies: individual: Mrk 231 - galaxies: active - galaxies: evolution - quasars: general - ISM: jets and outflows ISM: molecules

\section{Introduction}

Outflows driven by active galactic nuclei (AGNs) and/or starbursts, which may clear central regions of interstellar gas within a few tens of Myr, are a strong and direct mechanism for feedback in galaxies. Many galactic winds and outflows drive out large amounts of molecular gas (see e.g. Nakai et al. 1987; Walter et al. 2002; Sakamoto et al. 2006; Tsai et al. 2009; Alatalo et al. 2011; Sturm et al. 2011; Aalto et al. 2012b; Bolatto et al. 2013) and the ultimate fate of the expelled cold gas is not understood. It is also not clear if the molecular gas is formed in the outflow itself, or if it is carried out from the disc in molecular form. Studying the physical and chemical conditions of the outflowing molecular gas will help us understand the driving mechanism, the origin of the gas, and how it evolves in the wind.

\footnotetext{
* Based on observations with the IRAM Plateau de Bure Interferometer. IRAM is supported by INSU/CNRS (France), MPG (Germany), and IGN (Spain).

$\star \star$ Reduced datacubes as FITS files are only available at the CDS via anonymous ftp to cdsarc.u-strasbg. fr (130.79.128.5) or via http://cdsarc.u-strasbg.fr/viz-bin/qcat?]/A+A/587/A15 $\star \star \star$ NASA Postdoctoral Program Fellow.
}

The ultra-luminous infrared galaxy (ULIRG) Mrk 231 $\left(\log \left(L_{\mathrm{IR}} / L_{\odot}\right)=12.37\right)$, often referred to as the most nearby $(175 \mathrm{Mpc})$ quasi-stellar object (quasar), is a major galaxy merger and hosts both AGN activity and a young, dusty starburst with an extreme star formation rate of $\approx 200 M_{\odot} \mathrm{yr}^{-1}$ (Taylor et al. 1999; Gallagher et al. 2002; Lipari et al. 2009). Mrk 231 is well known for its massive molecular outflow (Feruglio et al. 2010; Fischer et al. 2010). The outflow rate of molecular gas is estimated to $700 M_{\odot} \mathrm{yr}^{-1}$ (Feruglio et al. 2010), which could empty the reservoir of molecular gas within $10 \mathrm{Myr}$. Alatalo (2015) estimates that only $\sim 200 M_{\odot} \mathrm{yr}^{-1}$ of the molecular gas actually escapes the system, which gives a depletion timescale of 50 Myr. The high-mass outflow rates originating in an extremely compact nuclear region $(\sim 0.01 \mathrm{pc}$; Feruglio et al. 2010 , $2015)$ and the wide-angle outflow of neutral atomic gas ( $\sim 3 \mathrm{kpc}$; Rupke \& Veilleux 2011) both seem to support the notion of an AGN driven outflow. Furthermore, through far-infrared $\mathrm{OH}$ observations González-Alfonso et al. (2014) detected two main outflow components in Mrk 231: a high-velocity component $\left(\lesssim 1500 \mathrm{~km} \mathrm{~s}^{-1}\right.$ ) with highly excited $\mathrm{OH}$ emission, indicating that this gas is generated in a compact nuclear region of the galaxy, and a less excited low-velocity component $\left(\lesssim 600 \mathrm{~km} \mathrm{~s}^{-1}\right)$ 
representing more spatially extended outflowing gas. Teng et al. (2014) reported that Mrk 231 has an unusually low X-ray luminosity relative to its bolometric luminosity, and argue that this is a result of super-Eddington accretion in the galactic nucleus.

In previous IRAM Plateau de Bure Interferometer (PdBI) observations, Aalto et al. (2012a) found an extremely high $\mathrm{HCN} / \mathrm{CO} 1-0$ line ratio (0.3-1) in the Mrk 231 outflow, which suggests that a large fraction of the molecular gas in the outflow is dense $\left(n \gtrsim 10^{4} \mathrm{~cm}^{-3}\right)$. Similar HCN/CO line ratios are found in the outflows of M51; it has been suggested that these ratios are caused by shocks in the outflow (Matsushita et al. 2015). HCN has also been detected in the low-velocity molecular outflow of the Seyfert galaxy NGC 1068 (García-Burillo et al. 2014). Recently we reported HCN 3-2/1-0 line ratios in Mrk 231, which suggest that the emission is emerging from gas of $n \approx 4-$ $5 \times 10^{5} \mathrm{~cm}^{-3}$ with a high $\mathrm{HCN}$ abundance $\left(X(\mathrm{HCN}) \approx 10^{-8}\right.$ $10^{-6}$ ) and with upper limits of the mass and momentum flux of $4 \times 10^{8} M_{\odot}$ and $12 L_{\mathrm{AGN}} / c$, respectively (Aalto et al. 2015a). The precise structure and driving mechanism of the outflow is, however, still unclear. Among the suggested interpretations are acceleration by the radio jet and boosting of the molecular outflow by an ultra-fast outflow (UFO), i.e. a nuclear semi-relativistic wind (Feruglio et al. 2015), or (in general) radiation pressure on dust grains (see e.g. Murray et al. 2005).

In this paper we present IRAM PdBI $3 \mathrm{~mm}$ and $2 \mathrm{~mm}$ data of the $\mathrm{HCN}$ and $\mathrm{HCO}^{+}$outflow emission in Mrk 231. We also combine these data with previously published $3 \mathrm{~mm}$ and $1 \mathrm{~mm}$ PdBI data (Aalto et al. 2012a, 2015a). We can show for the first time that the emission from $\mathrm{HCN}, \mathrm{HCO}^{+}$, and $\mathrm{HNC}$ peaks at different velocities, implying chemical differentiation in the outflow. We interpret this as a result of shocks in the outflow and discuss it in relation to its driving mechanism.

\section{Observations}

We have used observations of the HCN 1-0 and 2-1 lines in Mrk 231 carried out with the six-element IRAM PdBI between May 2012 and January 2013. The WideX correlator was configured to cover a bandwidth of $3.6 \mathrm{GHz}$ centred at $84.1 \mathrm{GHz}$ and $170.5 \mathrm{GHz}$, respectively. We adopt a redshift of $z=$ 0.042170 (heliocentric frame) for Mrk 231 (Carilli et al. 1998). In the final spectra, the $1-0$ data were smoothed to $\sim 70 \mathrm{~km} \mathrm{~s}^{-1}$ and the 2-1 data to $\sim 40 \mathrm{~km} \mathrm{~s}^{-1}$.

For the $\mathrm{HCN} 2-1$ tuning, which includes the $\mathrm{HCO}^{+} 2-1$ line, the configuration was compact (PdBI D array); the shortest projected baseline was $18 \mathrm{~m}$. This corresponds to a maximum recoverable scale of $12^{\prime \prime}$. For the HCN 1-0 tuning, which included $\mathrm{HCO}^{+} 1-0$, the configuration was extended (A array); the shortest projected baseline was $150 \mathrm{~m}$, corresponding to maximum recoverable scales of $3^{\prime \prime}$. However, the $\mathrm{HCN}$ and $\mathrm{HCO}^{+} 1-0$ data (including their wide linewings) were combined with data previously obtained in a more compact PdBI configuration (B array; Aalto et al. 2012a) with a maximum recoverable scale of $6^{\prime \prime}$. This scale is much larger than the extent of the source in both the line core and the wings, so we do not expect any significant loss of flux. The resulting synthesised beams are between 1-2" depending on the lines. The combination of the two 1-0 data sets was executed using the CASA task clean and the weights were re-evaluated, normalised by the number of measurement points.

The data reduction was done with the GILDAS/CLIC ${ }^{1}$ package in a standard way. The bandpass response was calibrated

\footnotetext{
1 GILDAS is developed by the IRAM institute, Grenoble, France, and can be downloaded from http: //wWw . iram. fr/IRAMFR/GILDAS
}

by observing bright radio quasars. The flux calibration was done with MWC349, using the flux model in GILDAS, or the quasar $1150+497$. The amplitude and phase gains were derived from the quasar $1150+497$. After calibration the data were exported into $\mathrm{CASA}^{2}$ for imaging and analysis. We adopt a natural weighting scheme. Details on the observing dates, resulting rms levels, and synthesised beam sizes are given in Table 1 .

In this work, we also use $1 \mathrm{~mm}$ PdBI A-array and B-array data of the $\mathrm{HCN}$ and $\mathrm{HCO}^{+} 3-2$ transitions previously reported by Aalto et al. (2015a), and $3 \mathrm{~mm}$ PdBI B-array observations of the HNC 1-0 transition (Aalto et al. 2012a).

\section{Results}

\subsection{Continuum emission}

The continuum emission detected in the $3 \mathrm{~mm} \mathrm{~A}$-array data peaks at the position $\alpha=12: 56: 14.23, \delta=+56: 52: 25.2$ (J2000). The $3 \mathrm{~mm}(88 \mathrm{GHz})$ continuum emission in this data set (observed in January 2013) was extracted in channels free from line emission. The continuum level was then estimated through a Gaussian fit of the image plane data. The continuum level was found to be $50.7 \pm 0.4 \mathrm{mJy}$, which is considerably higher than was found in the older $3 \mathrm{~mm}(89 \mathrm{GHz}) \mathrm{B}$-array observations: $25.0 \pm 0.6 \mathrm{mJy}$ (Aalto et al. 2012a, observed in March 2011). During the time period in question, Mrk 231 was undergoing a flare event. Through VLA observations, Reynolds et al. (2013) found that the $20 \mathrm{GHz}$ continuum flux of Mrk 231 increased by roughly a factor of 2 in this time period. Aalto et al. (2015a) also found an increase in the $1 \mathrm{~mm}(256 \mathrm{GHz})$ continuum flux from 24 mJy to 44 mJy between February 2012 and February 2013, which is consistent with the $20 \mathrm{GHz}$ observations of Reynolds et al. (2013).

\subsection{Molecular line emission}

The $3 \mathrm{~mm}$ and $2 \mathrm{~mm}$ spectra at the peak of the continuum emission are shown in Fig. 1 . In the A-array $3 \mathrm{~mm}$ data we detect spectral lines of $\mathrm{HCN}, \mathrm{HCO}^{+}, \mathrm{SiO}, \mathrm{CCH}, \mathrm{H}^{13} \mathrm{CN}, \mathrm{HC}^{15} \mathrm{~N}$, and $\mathrm{H}^{13} \mathrm{CO}^{+}$. In the D-array $2 \mathrm{~mm}$ data we detect the $\mathrm{HCN}, \mathrm{HCO}^{+}$, and $\mathrm{HOC}^{+} 2-1$ spectral lines. The $\mathrm{HCN}$ and $\mathrm{HCO}^{+} 1-0$ and $2-$ 1 lines show very wide line wings with a full width at zero intensity of $1500-2000 \mathrm{~km} \mathrm{~s}^{-1}$. The $\mathrm{HCN}$ and $\mathrm{HCO}^{+} 1-0$ lines are also covered in PdBI B-array observations (Aalto et al. 2012a), and we combined the two $3 \mathrm{~mm}$ data sets to acquire a higher $(u, v)$ coverage and signal-to-noise ratio $(\mathrm{S} / \mathrm{N})$. The $\mathrm{HCN}$ and $\mathrm{HCO}^{+}$lines have somewhat higher flux densities in the B-array data than in the A-array data owing to some extended emission being resolved out by the extended A-array configuration.

Table 2 lists all the detected spectral lines in the $3 \mathrm{~mm}$ and $2 \mathrm{~mm}$ observations, and Table 3 lists the extracted fluxes and fitted line parameters. Here we focus on the $\mathrm{HCN}$ and $\mathrm{HCO}^{+}$observations.

Below, we define the line core emission as any emission between $-250 \mathrm{~km} \mathrm{~s}^{-1}$ and $250 \mathrm{~km} \mathrm{~s}^{-1}$ with respect to the systemic velocity of Mrk 231. Following the Aalto et al. (2012a) definition in Mrk 231, outflow (line wing) emission is any emission between $\pm 350 \mathrm{~km} \mathrm{~s}^{-1}$ and $\pm 990 \mathrm{~km} \mathrm{~s}^{-1}$.

2 The Common Astronomy Software Applications package can be acquired from http://casa.nrao.edu/ 
Table 1. Observational details.

\begin{tabular}{|c|c|c|c|c|c|c|}
\hline Obs. window & $\begin{array}{l}\text { Frequency range }^{a} \\
\text { [GHz] }\end{array}$ & $\begin{array}{l}\mathrm{rms}^{b} \\
{\left[\mathrm{mJy}\left(\mathrm{km} \mathrm{s}^{-1}\right)^{-1}\right]}\end{array}$ & Obs. date & $\begin{array}{l}\text { Synth. beam } \\
{\left[{ }^{\prime \prime} \times\right]}\end{array}$ & $\begin{array}{l}\mathrm{PA} \\
{\left[{ }^{\circ}\right]}\end{array}$ & Array \\
\hline $3 \mathrm{~mm}$ & $85.787-89.520$ & 2.50 & Jan. 2013 & $0.95 \times 0.80$ & -8 & $\mathrm{~A}$ \\
\hline $3 \mathrm{~mm}$ & $87.991-89.520$ & 2.03 & Mar. 2011 \& Jan. 2013 & $1.15 \times 0.97$ & 33 & $\mathrm{~A}+\mathrm{B}^{c}$ \\
\hline $2 \mathrm{~mm}$ & $175.817-179.554$ & 5.21 & May-Oct. 2012 & $2.89 \times 1.99$ & -62 & $\mathrm{D}$ \\
\hline
\end{tabular}

Notes. ${ }^{(a)}$ Rest frequencies assuming the redshift of $z=0.042170$ for Mrk 231 (Carilli et al. 1998). ${ }^{(b)}$ Typical rms per beam per km s${ }^{-1}$ in line-free parts of the spectra. ${ }^{(c)}$ Combination of the A-array data (above) and B-array data previously reported by Aalto et al. (2012a).
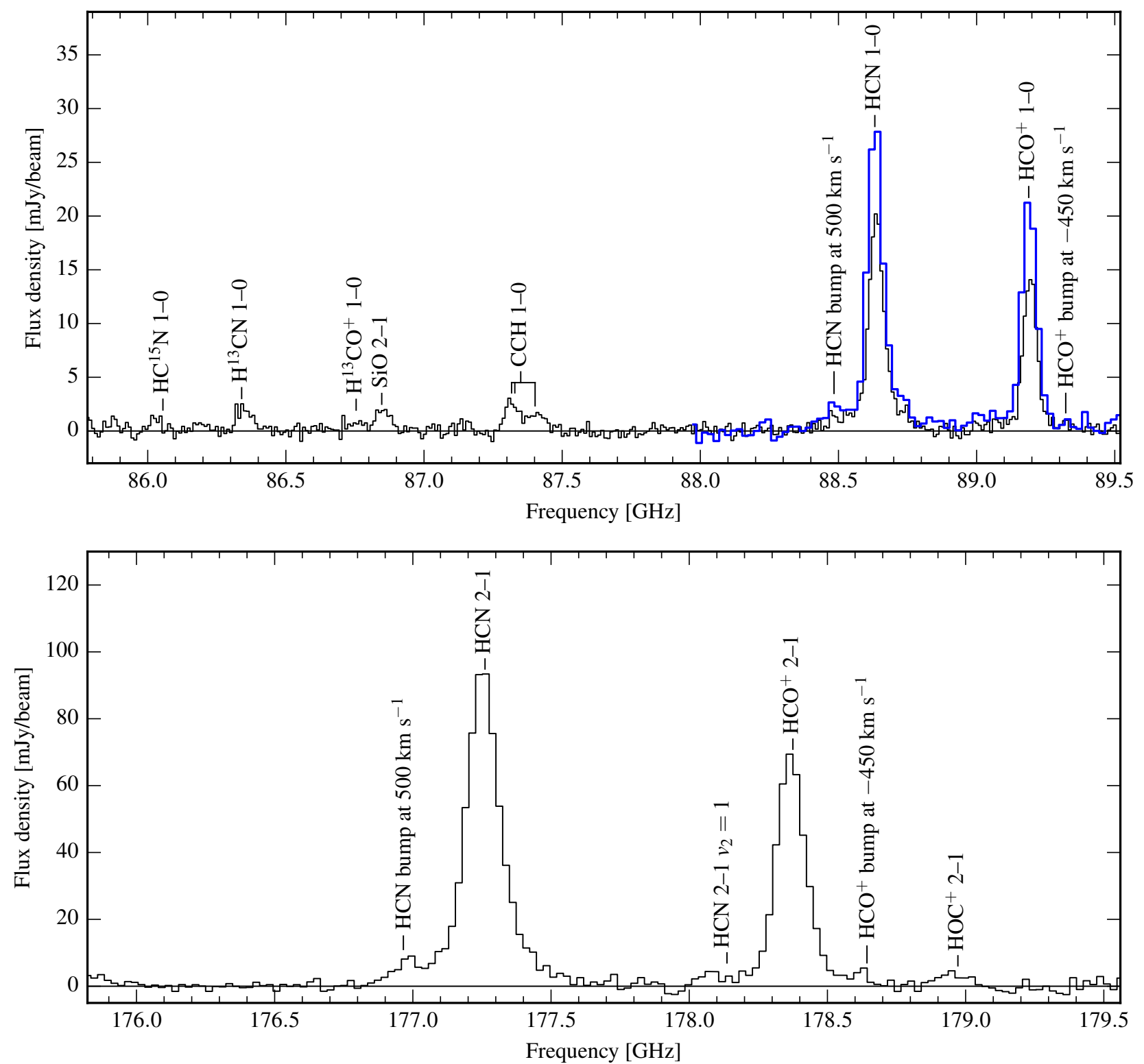

Fig. 1. Top: $3 \mathrm{~mm}$ A-array (black line) and B-array (thick blue line; Aalto et al. 2012a) spectra of Mrk 231 towards the central beam. Bottom: $2 \mathrm{~mm}$ D-array spectrum. The frequency of the HCN 2-1 $v_{2}=1$ line is indicated in the plot, but this line is not significantly detected. Both figures: the most prominent outflow bumps of $\mathrm{HCN}$ and $\mathrm{HCO}^{+}$emission are also indicated. The frequency axes of the two spectra are corrected for the redshift of the source $(z=0.042170)$. The beam sizes can be found in Table 1 .

\subsection{1. $\mathrm{HCN}$}

Outflow. As previously reported (Aalto et al. 2012a) and also seen in CO data (Cicone et al. 2012), the HCN 1-0 line shows emission at velocities $>350 \mathrm{~km} \mathrm{~s}^{-1}$ from the line centre, which indicates a dense molecular outflow. The red component is more prominent at higher velocities than the blue component. As indicated in Fig. 1, the HCN 1-0 and 2-1 lines show high-velocity bumps, the most prominent at $+500 \mathrm{~km} \mathrm{~s}^{-1}$.

Line core. We detect the $\mathrm{HCN}$ isotopologues $\mathrm{H}^{13} \mathrm{CN}$ and $\mathrm{HC}^{15} \mathrm{~N}$. Costagliola et al. (2011) have previously detected $\mathrm{H}^{13} \mathrm{CN}$ in Mrk 231, but $\mathrm{HC}^{15} \mathrm{~N}$ was detected for the first time in this galaxy. 
Table 2. Detected spectral lines.

\begin{tabular}{llcc}
\hline \hline Species & Transition & $\begin{array}{l}\text { Frequency }^{a} \\
{[\mathrm{GHz}]}\end{array}$ & $\begin{array}{l}E_{\mathrm{u}} / k^{a} \\
{[\mathrm{~K}]}\end{array}$ \\
\hline $\mathrm{HC}^{15} \mathrm{~N}$ & $J=1 \rightarrow 0$ & 86.05497 & 4.13 \\
$\mathrm{H}^{13} \mathrm{CN}$ & $J=1 \rightarrow 0$ & 86.33992 & 4.14 \\
$\mathrm{H}^{13} \mathrm{CO}^{+}$ & $J=1 \rightarrow 0$ & 86.75429 & 4.16 \\
$\mathrm{SiO}$ & $J=2 \rightarrow 1$ & 86.84696 & 6.25 \\
$\mathrm{CCH}$ & $N=1 \rightarrow 0^{b}$ & 87.31690 & 4.19 \\
$\mathrm{CCH}$ & $N=1 \rightarrow 0^{c}$ & 87.32859 & 4.19 \\
$\mathrm{CCH}$ & $N=1 \rightarrow 0^{d}$ & 87.40199 & 4.20 \\
$\mathrm{HCN}$ & $J=1 \rightarrow 0$ & 88.63160 & 4.25 \\
$\mathrm{HCO}^{+}$ & $J=1 \rightarrow 0$ & 89.18852 & 4.28 \\
\hline $\mathrm{HCN}$ & $J=2 \rightarrow 1$ & 177.26111 & 12.76 \\
$\mathrm{HCO}^{+}$ & $J=2 \rightarrow 1$ & 178.37506 & 12.84 \\
$\mathrm{HOC}^{+}$ & $J=2 \rightarrow 1$ & 178.97205 & 12.88 \\
\hline
\end{tabular}

Notes. ${ }^{(a)}$ Rest frequencies and energy levels from the CDMS database (Müller et al. 2001). ${ }^{(b)} J=3 / 2 \rightarrow 1 / 2, F=2 \rightarrow 1$. $^{(c)} J=3 / 2 \rightarrow 1 / 2$, $F=1 \rightarrow 0 .{ }^{(d)} J=1 / 2 \rightarrow 1 / 2, F=1 \rightarrow 1$.

The $\mathrm{H}^{13} \mathrm{CN} / \mathrm{HC}^{15} \mathrm{~N}$ line ratio is $2.4 \pm 0.6$, which is lower than the $\mathrm{H}^{13} \mathrm{CN} / \mathrm{HC}^{15} \mathrm{~N}$ line ratio found in NGC 4418 (3.7; Sakamoto et al. 2010), but higher than in IC 694 (1.2; Jiang et al. 2011) and NGC 4945 (0.96; Wang et al. 2004).

Aalto et al. (2015a) have detected gas on forbidden velocities westwards of the central peak along the EW axis by the use of a PV diagram of the HCN 3-2 emission. In this context, forbidden velocities means that this emission does not represent an extension of the rotating disc; in this case the gas is $50-100 \mathrm{~km} \mathrm{~s}^{-1}$ too fast. This could for instance represent gas in a bar structure, an inflow, or some kind of foreground gas. This emission could also represent low-velocity outflowing gas displaced by shocks. Instead, the $\mathrm{HCO}^{+}$emission lines have no detectable signal at corresponding positions and velocities.

\subsection{2. $\mathrm{HCO}^{+}$and $\mathrm{HOC}^{+}$}

Outflow. Like the $\mathrm{HCN}$ lines, the $\mathrm{HCO}^{+}$lines show emission at outflow velocities, although fainter than is detected in HCN. The $\mathrm{HCO}^{+} 1-0$ and $2-1$ lines show high-velocity bumps (Fig. 1) as the $\mathrm{HCN}$ lines do, but the most apparent $\mathrm{HCO}^{+}$bump appears at a lower velocity of approximately $-450 \mathrm{~km} \mathrm{~s}^{-1}$.

Line core. We also detect the $\mathrm{H}^{13} \mathrm{CO}^{+} 1-0$ line and the $\mathrm{HOC}^{+} 2-1$ line. The $\mathrm{HCO}^{+} / \mathrm{H}^{13} \mathrm{CO}^{+} 1-0$ ratio is $17 \pm 4$, whereas the $\mathrm{HCN} / \mathrm{H}^{13} \mathrm{CN} 1-0$ ratio is only $10 \pm 1$ (both calculated for the A-array data, since the ${ }^{13} \mathrm{C}$-species are not covered in the B-array observations), indicating a high optical depth of the HCN line core. We find that the $\mathrm{HCO}^{+} / \mathrm{HOC}^{+} 2-1$ line ratio is only $19 \pm 3$, which is even lower than the value found in the nearby AGN NGC $1068\left(\mathrm{HCO}^{+} / \mathrm{HOC}^{+} 1-0 \approx 40\right.$; Usero et al. 2004), which possibly indicates strong XDR and/or PDR activity in Mrk 231. However, since this is calculated from the possibly optically thick $\mathrm{HCO}^{+} 2-1$ line, the line ratio might be underestimating the abundance ratio.

The $\mathrm{HCO}^{+}$lines are 10-20\% narrower than the $\mathrm{HCN}$ lines. No emission at forbidden velocities is found in connection with the line core emission (see also Sect. 4.1).

\section{Discussion}

\subsection{Line core emission}

We define line core emission in Mrk 231 as any emission between $-250 \mathrm{~km} \mathrm{~s}^{-1}$ and $250 \mathrm{~km} \mathrm{~s}^{-1}$, assuming that this mainly originates in non-outflowing gas.

We normalised the HCN 1-0, 2-1, and 3-2 lines; the $\mathrm{HCO}^{+} 1-0$ and $2-1$ line profiles; and the $\mathrm{HNC} 1-0$ line to unity. The $\mathrm{HCO}^{+} 3-2$ line was excluded owing to contamination from the HCN 3-2 $v_{2}=1$ line (see below). The normalised HCN and $\mathrm{HCO}^{+}$lines were then stacked (weighted by their $\mathrm{S} / \mathrm{N}$ ) to allow for a higher $\mathrm{S} / \mathrm{N}$ comparison of the molecular emission velocity profiles. The result is shown in Fig. 2; it is clear that the HCN emission has a broader core component than the two other species. This extra emission could have the same origin as the forbidden velocity component seen in the HCN 3-2 PV diagram reported by Aalto et al. (2015a), representing gas that is not an extension of the rotating disc. However, it could also be explained by high optical depth or possibly even selfabsorption of the $\mathrm{HCN}$ line emission (as appears likely from the observed $\mathrm{HCN} / \mathrm{H}^{13} \mathrm{CN}$ and $\mathrm{HCO}^{+} / \mathrm{H}^{13} \mathrm{CO}^{+}$ratios; see above), which causes a slightly broader line profile. Other (U)LIRGs have shown a very high rate of self-absorption of the HCN lines (Aalto et al. 2015b).

\subsection{Line wing emission}

In the discussion below, we follow the definition of line wings in Mrk 231 from Aalto et al. (2012a), who define the red line wing as gas with velocities between $350 \mathrm{~km} \mathrm{~s}^{-1}$ and $990 \mathrm{~km} \mathrm{~s}^{-1}$, and the blue line wing between $-990 \mathrm{~km} \mathrm{~s}^{-1}$ and $-350 \mathrm{~km} \mathrm{~s}^{-1}$, with respect to the systemic velocity. This emission mainly originates in an almost face-on outflow. We only detect such wings in the strongest spectral lines, those of the main isotopologues of $\mathrm{HCN}$, $\mathrm{HCO}^{+}$, and $\mathrm{HNC}$. Outflow line wings have also been detected in Mrk 231 through CO observations (Cicone et al. 2012).

We make a first attempt to study any difference between the $\mathrm{HCN}, \mathrm{HCO}^{+}$, and $\mathrm{HNC}$ distributions by investigating the ratio of the normalised and stacked $\mathrm{HCN}, \mathrm{HCO}^{+}$, and $\mathrm{HNC}$ line profiles in Fig. 2. Figure 3 shows the $\mathrm{HCN} / \mathrm{HCO}^{+}$and $\mathrm{HCN} / \mathrm{HNC}$ ratios as a function of velocity. At low $\left(<150 \mathrm{~km} \mathrm{~s}^{-1}\right)$ absolute velocities, an increasing optical depth of HCN towards the line centre becomes apparent, but at higher velocities we notice a highly variable and symmetric $\mathrm{HCN} / \mathrm{HCO}^{+}$ratio, oscillating between 1 and 2.5 times the ratio at $0 \mathrm{~km} \mathrm{~s}^{-1}$. The $\mathrm{HCN} / \mathrm{HNC}$ ratio also shows considerable variation between 1 and $\sim 8$ times the ratio at $0 \mathrm{~km} \mathrm{~s}^{-1}$.

\subsubsection{Outflow clumps}

In Fig. 1, we found that the HCN 1-0 and 2-1 lines both have outflow features at $500 \mathrm{~km} \mathrm{~s}^{-1}$, whereas the $\mathrm{HCO}^{+} 1-0$ and 2-1 lines have features at $-450 \mathrm{~km} \mathrm{~s}^{-1}$. In Figs. 2-4, this difference between $\mathrm{HCN}$ and $\mathrm{HCO}^{+}$can be seen more clearly.

To search for further irregularities of this kind, we made $\mathrm{PV}$ diagrams of the $\mathrm{HCN}, \mathrm{HCO}^{+}$, and $\mathrm{HNC}$ emission (Figs. A.1 and A.2). The HCN $1-0$ and 2-1 PV diagrams agree well to a first order with the HCN 1-0 and 3-2 PV diagrams presented by Aalto et al. (2012a, 2015a). In particular, several spectral features at high velocities and small position offsets are detected. We also detect several similar features in the $\mathrm{HCO}^{+}$and $\mathrm{HNC}$ PV diagrams. The strongest outflow features are found at approximately $500 \mathrm{~km} \mathrm{~s}^{-1}, 350 \mathrm{~km} \mathrm{~s}^{-1}$, and $-400 \mathrm{~km} \mathrm{~s}^{-1}$ for $\mathrm{HCN}$ 
Table 3. Fitted line parameters towards the continuum peak position.

\begin{tabular}{|c|c|c|c|c|c|c|c|}
\hline \multirow[b]{2}{*}{ Species } & \multirow[b]{2}{*}{ Transition } & \multirow[b]{2}{*}{$\begin{array}{l}F W H M \\
{\left[\mathrm{~km} \mathrm{~s}^{-1}\right]}\end{array}$} & \multicolumn{2}{|c|}{ Core } & \multicolumn{2}{|c|}{ Wings, peak int. intensity } & \multirow[b]{2}{*}{$\begin{array}{l}\text { Array } \\
\text { configuration(s) }\end{array}$} \\
\hline & & & $\begin{array}{l}\text { Peak flux } \\
{\left[\mathrm{mJy} \mathrm{beam}^{-1}\right]}\end{array}$ & $\begin{array}{l}\text { Peak int. intensity }{ }^{a} \\
{\left[\mathrm{Jy} \mathrm{beam}^{-1} \mathrm{~km} \mathrm{~s}^{-1}\right]}\end{array}$ & $\begin{array}{l}\text { Red wing }^{b} \\
\quad\left[\mathrm{Jy} \mathrm{beam}^{-}\right.\end{array}$ & $\begin{array}{l}\text { Blue wing }{ }^{c} \\
\left.-1 \mathrm{~km} \mathrm{~s}^{-1}\right]\end{array}$ & \\
\hline $\mathrm{HC}^{15} \mathrm{~N}$ & $J=1 \rightarrow 0$ & 151 & 1.29 & $0.22 \pm 0.05$ & $\ldots$ & $\ldots$ & A \\
\hline $\mathrm{H}^{13} \mathrm{CN}$ & $J=1 \rightarrow 0$ & 226 & 2.17 & $0.50 \pm 0.05$ & $\ldots$ & $\ldots$ & A \\
\hline $\mathrm{H}^{13} \mathrm{CO}^{+}$ & $J=1 \rightarrow 0$ & 258 & 0.77 & $0.18 \pm 0.04$ & $\begin{array}{l}\cdots \\
\cdots\end{array}$ & $\begin{array}{l}\cdots \\
\cdots\end{array}$ & A \\
\hline $\mathrm{SiO}$ & $J=2 \rightarrow 1$ & 207 & 2.06 & $0.45 \pm 0.05$ & $\ldots$ & $\ldots$ & A \\
\hline $\mathrm{CCH}$ & $N=1 \rightarrow 0^{d}$ & 172 & 2.73 & & & & A \\
\hline $\mathrm{CCH}$ & $N=1 \rightarrow 0^{e}$ & 314 & 1.58 & $0.99 \pm 0.07$ & $\cdots$ & $\cdots$ & A \\
\hline $\mathrm{HCN}$ & $J=1 \rightarrow 0$ & 217 & 24.5 & $5.76 \pm 0.05$ & $0.54 \pm 0.05$ & $0.46 \pm 0.05$ & $\mathrm{~A}+\mathrm{B}$ \\
\hline $\mathrm{HCO}^{+}$ & $J=1 \rightarrow 0$ & 197 & 18.5 & $3.95 \pm 0.05$ & $0.38 \pm 0.05$ & $0.21 \pm 0.05$ & $\mathrm{~A}+\mathrm{B}$ \\
\hline $\mathrm{HCN}$ & $J=2 \rightarrow 1$ & 255 & 90.6 & $24.09 \pm 0.14$ & $1.74 \pm 0.14$ & $1.18 \pm 0.14$ & $\mathrm{D}$ \\
\hline $\mathrm{HCO}^{+}$ & $J=2 \rightarrow 1$ & 215 & 68.0 & $15.58 \pm 0.14$ & $0.78 \pm 0.14$ & $1.09 \pm 0.14^{f}$ & $\mathrm{D}$ \\
\hline $\mathrm{HOC}^{+}$ & $J=2 \rightarrow 1$ & 212 & 3.88 & $0.82 \pm 0.12$ & $\ldots$ & $\ldots$ & $\mathrm{D}$ \\
\hline
\end{tabular}

Notes. ${ }^{(a)}$ Integrated intensity. The $\mathrm{HCN}$ and $\mathrm{HCO}^{+}$lines are integrated between -250 and $250 \mathrm{~km} \mathrm{~s}^{-1}$. $^{(b)}$ Integrated between 350 and $990 \mathrm{~km} \mathrm{~s}^{-1}$ towards the continuum peak position. ${ }^{(c)}$ Integrated between -990 and $-350 \mathrm{~km} \mathrm{~s}^{-1}$ towards the continuum peak position. ${ }^{(d)} \mathrm{Blend}$ of $J=3 / 2 \rightarrow 1 / 2$, $F=2 \rightarrow 1$ and $J=3 / 2 \rightarrow 1 / 2, F=1 \rightarrow 0$ lines. ${ }^{(e)} J=1 / 2 \rightarrow 1 / 2, F=1 \rightarrow 1$ line. ${ }^{(f)}$ Blended by the HOC $^{+} 2 \rightarrow 1$ line.

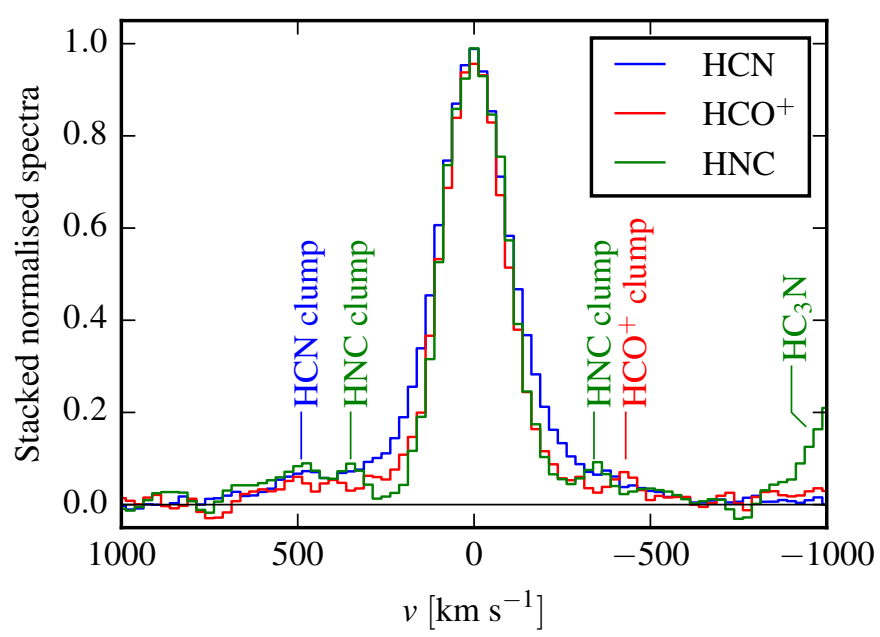

Fig. 2. $\mathrm{HCN} 1-0,2-1$, and 3-2 lines; the $\mathrm{HCO}^{+} 1-0$ and 2-1 lines; and the HNC 1-0 line, all measured towards the central beam, are shown normalised, regridded, and stacked weighted by the $\mathrm{S} / \mathrm{N}$ to show the variations in line profile between the species. The $\mathrm{HCO}^{+} 3-2$ line was not included to avoid contamination from the HCN 3-2 $v_{2}=1$ line. Some of the more important outflow clumps are indicated.

and $400 \mathrm{~km} \mathrm{~s}^{-1}$ and $-450 \mathrm{~km} \mathrm{~s}^{-1}$ for $\mathrm{HCO}^{+}$. Most of these features are in good agreement with the features in the spectra in Sect. 3, but the faintest features cannot be seen in the spectra.

To check for possible contamination of the outflow spectral features, we used the spectral line database Splatalogue ${ }^{3}$ to search for spectral lines with frequencies matching these clumps. Aalto et al. (2015a) have detected the HCN 3-2 $v_{2}=1$ vibrational line in PdBI observations of Mrk 231. This line blends with the redshifted $\mathrm{HCO}^{+} 3-2$ outflow line wing and shows up as a feature seen at $400 \mathrm{~km} \mathrm{~s}^{-1}$ in the $\mathrm{HCO}^{+} 3-2$ line (see Fig. 1 in Aalto et al. 2015a). However, the HCN 2-1 $v_{2}=1$ line is not detected in our data (see Fig. 1). This is in agreement with expectations from the HCN 3-2 $v_{2}=1$ line strength; assuming optically thin emission, the HCN 2-1 $v_{2}=1$ should have

\footnotetext{
3 http://www.splatalogue.net/
}

a line strength around $1.6 \mathrm{mJy}^{-1} \mathrm{bam}^{-1}$, about half of the measured flux density at that frequency. Thus, a fraction of the emission detected in the $\mathrm{HCO}^{+} 2-1$ red wing at around $400 \mathrm{~km} \mathrm{~s}^{-1}$ should originate in the HCN 2-1 $v_{2}=1$ line emission (which is not coincident with any of the outflow features). With the exception of the HCN vibrational lines, no likely blend candidates were found in Splatalogue for any of the three bands. The $\mathrm{HOC}^{+} 1-0,2-1$, and 3-2 lines should appear at $-1000 \mathrm{~km} \mathrm{~s}^{-1}$ in the corresponding $\mathrm{HCO}^{+}$lines (although the $\mathrm{HOC}^{+} 1-0$ line is not detected). This velocity is not consistent with any of the suggested clumps, but these lines must be considered when measuring the integrated flux of the blue line wings of $\mathrm{HCO}^{+}$. The lack of line-blend candidates in the database allows us to assume that all features detected in the outflow wings of the $\mathrm{HCO}^{+} 1-0$ and 2-1 lines at $v \gtrsim-820 \mathrm{~km} \mathrm{~s}^{-1}$, in the HNC line, and in all three HCN lines represent outflowing molecular gas. PdBI observations of the merging galaxy pair IRAS F08572+3915 show similar outflow features in a PV diagram of CO $1-0$ emission (Cicone et al. 2014).

To increase the $\mathrm{S} / \mathrm{N}$ of these clumps, we also stacked the $\mathrm{HCN} \mathrm{PV}$ diagrams and the $\mathrm{HCO}^{+} \mathrm{PV}$ diagrams. As above, the $\mathrm{HCO}^{+} 3-2$ data were not included owing to the HCN 3-2 $v_{2}=1$ blend. In the stacking, the combined $\mathrm{A}+\mathrm{B}$-array $\mathrm{PV}$ diagrams of the 1-0 and 3-2 lines and the D-array 2-1 PV diagram were used (see Appendix A). The stacking was weighted by the inverse of the rms of each set of observations. In the resulting stacked PV diagrams (Fig. 5), several significant features at high velocity and low position offset are seen. Since we find that the outflow features are amplified by this method it is even less plausible that blends from faint unidentified spectral lines are the cause of this emission.

Like in the spectra (Sect. 3), we find that the $\mathrm{HCN}$ and $\mathrm{HCO}^{+}$features appear at different velocities in the PV diagrams. The shifts in position between these features are all within errors of $0^{\prime \prime}$ offset. In Fig. 5, the features are illustrated by plus signs showing the $\mathrm{HCO}^{+}$features and crosses indicating the $\mathrm{HCN}$ features. For easier comparison, the symbols for both species are shown in all PV diagrams. In the HCN PV diagrams, the $\mathrm{HCN}$ markers are white and the $\mathrm{HCO}^{+}$markers are red. In the $\mathrm{HCO}^{+} \mathrm{PV}$ diagrams, the $\mathrm{HCO}^{+}$markers are white and the $\mathrm{HCN}$ markers are red. 

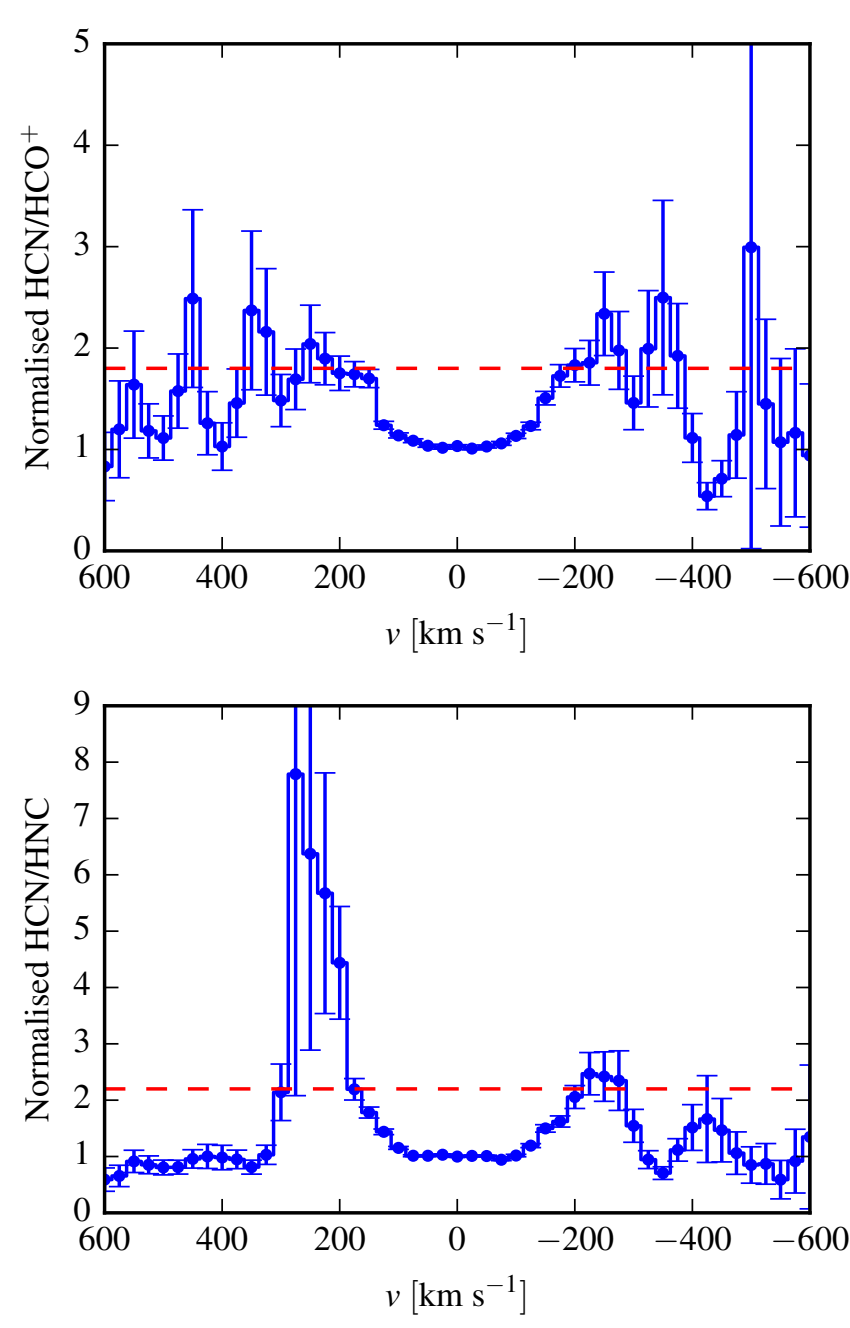

Fig. 3. Combined normalised $\mathrm{HCN}$ and $\mathrm{HCO}^{+}$line flux ratios (top panel) and the corresponding $\mathrm{HCN}$ and $\mathrm{HNC}$ ratios (bottom panel) from Fig. 2 are plotted as a function of velocity. Since the fluxes are normalised, the ratio is per definition 1 at $0 \mathrm{~km} \mathrm{~s}^{-1}$. Error bars are $1 \sigma$ (statistical errors only). The red dashed lines show the line core ratio assuming that both species are optically thin towards the edge of the line core (at $\pm 200 \mathrm{~km} \mathrm{~s}^{-1}$ ). We note that the $y$-axes of the graphs show neither line ratios nor abundance ratios.

Cicone et al. (2012) found a red outflow component of the CO 1-0 and 2-1 lines in PdBI observations of Mrk $231(v=$ $527 \pm 30 \mathrm{~km} \mathrm{~s}^{-1}, F W H M=276 \pm 71 \mathrm{~km} \mathrm{~s}^{-1}$ ). This is consistent with the strongest red clump found in our stacked HCN PV diagram, suggesting that the $\mathrm{CO}$ and $\mathrm{HCN}$ appear in the same clumps, displaced from the $\mathrm{HCO}^{+}$features.

The position of the outflow emission is consistent with the line core and continuum emission, also for the high-resolution 3-2 data (Aalto et al. 2015a). This shows that Mrk 231 has a face-on outflow, in agreement with the face-on disc reported by Bryant \& Scoville (1996) and Downes \& Solomon (1998), and also consistent with the blazar properties as shown by continuum observations (see Sect. 4.3).

As seen in Fig. 2, the HNC 1-0 outflow emission (Aalto et al. 2012a) also has features at different velocities from $\mathrm{HCN}$ and $\mathrm{HCO}^{+}$(see Fig. A.2), but the S/N level of the HNC 1-0 PV diagram is too low to quantify this.

As a summary of our findings, the HCN outflow emission shows features at around $500 \mathrm{~km} \mathrm{~s}^{-1}, 350 \mathrm{~km} \mathrm{~s}^{-1}$, and

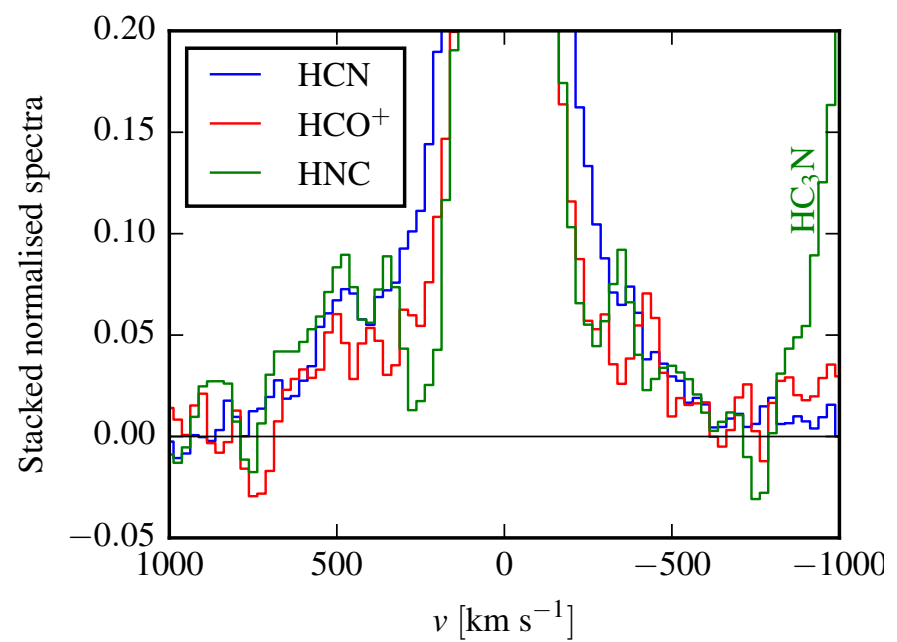

Fig. 4. Zoom-in of Fig. 2 showing the clumps of the separate species peaking at different velocities.

$-400 \mathrm{~km} \mathrm{~s}^{-1}$, while $\mathrm{HCO}^{+}$shows fainter features at $600 \mathrm{~km} \mathrm{~s}^{-1}$, $400 \mathrm{~km} \mathrm{~s}^{-1}$, and $-450 \mathrm{~km} \mathrm{~s}^{-1}$, and $\mathrm{HNC}$ has tentative features at $480 \mathrm{~km} \mathrm{~s}^{-1}, 350 \mathrm{~km} \mathrm{~s}^{-1}$ and $-340 \mathrm{~km} \mathrm{~s}^{-1}$. Thus, the outflow emission shows an offset in the peak velocities of these three species. The three species $\mathrm{HCN}, \mathrm{HNC}$, and $\mathrm{HCO}^{+}$all show features at different velocities in the red outflow. For the blue outflow, the $\mathrm{S} / \mathrm{N}$ level is lower, but tendencies towards differentiation can also be seen here.

\subsubsection{What causes the chemical differentiation?}

In Mrk 231, the most significant clump of each species appears in the same order in the red and the blue outflow $\left(\mathrm{HCO}^{+}\right.$ at $600 \mathrm{~km} \mathrm{~s}^{-1}$ and $-450 \mathrm{~km} \mathrm{~s}^{-1}$, $\mathrm{HCN}$ at $500 \mathrm{~km} \mathrm{~s}^{-1}$ and $-400 \mathrm{~km} \mathrm{~s}^{-1}$, and HNC at $350 \mathrm{~km} \mathrm{~s}^{-1}$ and $-340 \mathrm{~km} \mathrm{~s}^{-1}$ ). This apparent symmetry could be an actual effect of the structure and propagation of a shock, or it could be a geometric effect (e.g. an expanding nearly spherical shell). As the outflow appears to slow down with increasing radius, it suggests that the $\mathrm{HCO}^{+}$gas is enhanced close to the nucleus and HNC farthest out in the outflow. It should be noted, however, that this clump order is tentative since a number of fainter clumps are also identified.

Chemical differentiation in molecular outflows has previously been observed towards protostellar sources in the Milky Way (Tafalla et al. 2010; Tafalla \& Hacar 2013). As in Mrk 231, the $\mathrm{HCO}^{+}$component appears at a higher velocity than the HCN component in those protostellar outflows. Tafalla et al. note that the outflow component with the highest absolute velocity has a much lower $\mathrm{C} / \mathrm{O}$ ratio than the normal outflowing gas, suggesting that the high- and low-velocity components have different physical origins.

Since the $\mathrm{HCN} / \mathrm{HCO}^{+}$ratio is enhanced in shocks (Mitchell \& Deveau 1983), the HCN emission could trace the recently ejected gas which interacts with the surrounding gas in a shock. In the case of a decelerating outflow, and assuming the order of the strongest clumps above, $\mathrm{HNC}$ is primarily found in the slower post-shock gas, while $\mathrm{HCO}^{+}$is found in faster gas closer to the nucleus and before the shock (Podio et al. 2014 suggested that $\mathrm{HCO}^{+}$could be a tracer of pre-shocked gas in protostellar outflows). However, one complication is the comparison of $\mathrm{HCN}$ and $\mathrm{HNC}$ with $\mathrm{HCO}^{+}$, where the relative abundance will also be influenced by the $\mathrm{O} / \mathrm{N}$ abundances in the gas. 

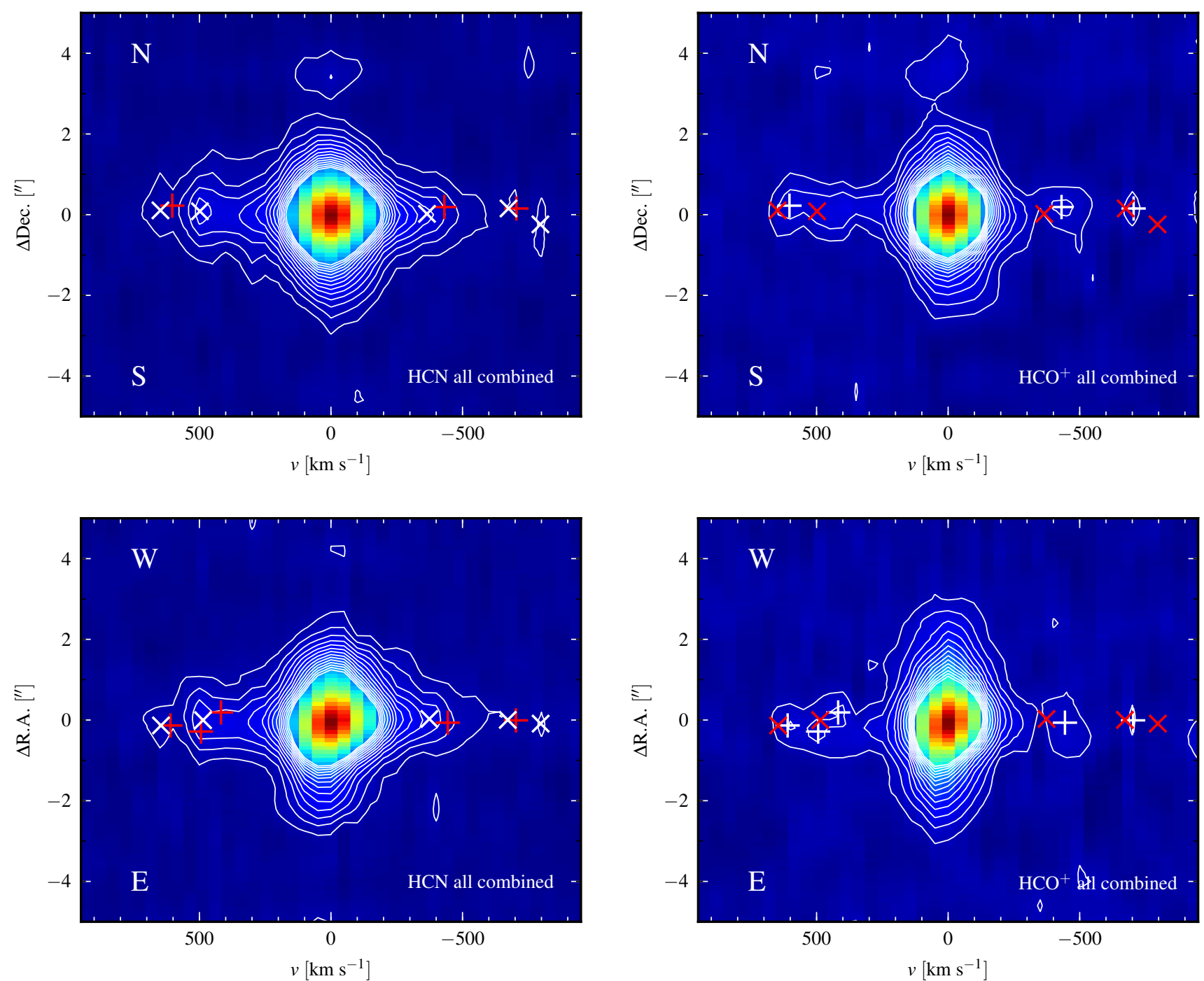

Fig. 5. Stacked PV diagrams of $\mathrm{HCN} 1-0,2-1$, and 3-2; and $\mathrm{HCO}^{+} 1-0$ and $2-1$ in the combined data (contours start at $2 \sigma$, and continue at $2 \sigma$ intervals). The $\mathrm{HCO}^{+} 3-2$ line is not included owing to the $\mathrm{HCN} \mathrm{3-2} v_{2}=1$ blend at $400 \mathrm{~km} \mathrm{~s}^{-1}$. In the PV diagrams, the positions of the $\mathrm{HCO}^{+}$outflow features identified in the stacked PV diagrams are indicated by plus signs and the HCN features are indicated by crosses. In the $\mathrm{HCN}$ PV diagrams, the $\mathrm{HCN}$ markers are white and the $\mathrm{HCO}^{+}$markers are red. In the $\mathrm{HCO}^{+} \mathrm{PV}_{\text {diagrams, the }} \mathrm{HCO}^{+}$markers are white and the HCN markers are red.

High-sensitivity PV diagrams of well-known shockenhanced species such as $\mathrm{SiO}, \mathrm{CH}_{3} \mathrm{OH}$, and $\mathrm{HNCO}$ will be important to test the hypothesis of shocks and their location in the Mrk 231 molecular outflow. In this scenario we would expect to see enhancements of shock species at velocities where $\mathrm{HCN}$ is peaking. To explain why the HNC gas appears at the lowest velocities, post-shock reformation of HNC must be investigated further.

Another possibility that could explain the observed chemical separation is that parts of the molecular outflow could be subject to different amounts of emission from the nuclear region as an effect of the physical structure of the nucleus and jet. In this scenario, it is interesting to note (again under the assumption of a decelerating outflow) that the $\mathrm{HCO}^{+}$enhancement is closest to the AGN.

\subsubsection{Radiative transfer modelling}

We have performed non-LTE radiative transfer modelling of the outflow emission (red and blue components of HCN, blue components of $\mathrm{HCO}^{+}$) with the RADEX radiative transfer code (van der Tak et al. 2007) to estimate the basic physical properties of the outflowing gas. We test the hypothesis of an outflow consisting of an ensemble of either self-gravitating clouds $(\Delta v=\sqrt{G M / R})$ or unbound clouds $(\Delta v \gg \sqrt{G M / R})$ as suggested by Aalto et al. (2015a), following the formalism and assumptions therein. We consider clouds with masses of $10 M_{\odot}$ and $T=50 \mathrm{~K}$ (Goldsmith 1987; see Aalto et al. 2015a for a discussion on these parameters). The self-gravitating cloud model should provide an upper limit of the outflow masses as the unbound cloud model will result in lower masses. We assume 10\% calibration errors on the integrated line fluxes in addition to the statistical errors. The $\mathrm{H}_{2}$ density and the absolute abundances of $\mathrm{HCN}$ and $\mathrm{HCO}^{+}$are set as free parameters. RADEX is used to calculate the line emission from a single clump. Both the modelled line emission from one clump and the observed line emission are then normalised to their respective $1-0$ line intensities (to account for the number of clumps), and the difference between these intensities is then minimised to find the best solution. In practice, this means that the number of clumps is the third free parameter. 

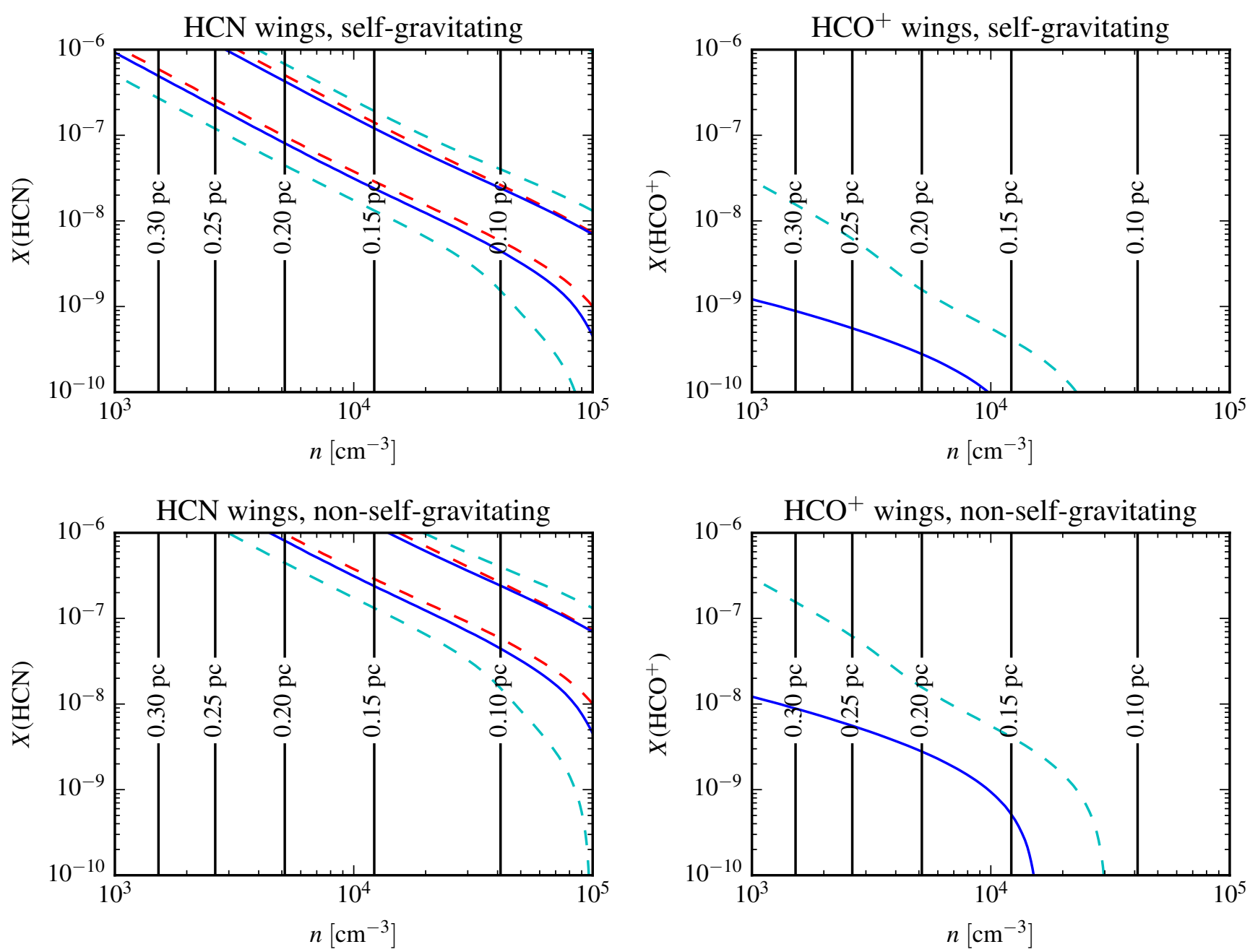

Fig. 6. Least- $\chi^{2}$ fits to the $\mathrm{HCN}$ and $\mathrm{HCO}^{+}$outflow emission using RADEX radiative-transfer modelling, assuming a clumpy outflow consisting of $10 M_{\odot}$ clouds at $T=50 \mathrm{~K}$. Red contours correspond to the red wing, and blue/cyan to the blue wing. Contours indicate $2 \sigma$ (solid blue) and $3 \sigma$ (dashed red and cyan) confidence levels. The black vertical lines show the radii of the individual clouds. For the $\mathrm{HCO}^{+}$, no red wing fits are made owing to contamination by vibrational HCN lines.

For $\mathrm{HCN}$, we measure the brightness temperature ratios in the red and blue line wings of the line emission towards the central beam, and find that the proportions are similar. The measured line ratios converted to brightness temperature scale are 1:0.75:0.25 for the transitions 1-0:2-1:3-2 (cf. Cols. 6 and 7 in Table 3). The $1-0 / 3-2$ ratio is slightly lower than the value 0.35 found by Aalto et al. (2015a), which is a result of the different methods of extracting this ratio. Our modelling shows that the observed intensities are consistent with cloud sizes of $0.3 \mathrm{pc}\left(n\left(\mathrm{H}_{2}\right) \approx 2 \times 10^{3} \mathrm{~cm}^{-3}\right)$ assuming $X(\mathrm{HCN})=10^{-6}$, and cloud sizes of $0.1 \mathrm{pc}\left(n\left(\mathrm{H}_{2}\right) \approx 4 \times 10^{4} \mathrm{~cm}^{-3}\right)$ if lowering the HCN abundance to $X(\mathrm{HCN})=10^{-8}$ (see the upper panels of Fig. 6), which is consistent with the results of Aalto et al. (2015a). We reach outflow masses $M_{\text {dense }}=3.3 \times 10^{8} M_{\odot}$ (both wings) for $X(\mathrm{HCN})=10^{-6}$ and $M_{\text {dense }}=1.4 \times 10^{9} M_{\odot}$ (both wings) for $X(\mathrm{HCN})=10^{-8}$, which are in good agreement with the values calculated by Aalto et al. (2015a), but they should be seen as upper limits of the outflow mass since here we assume self-gravitating clumps.

For $\mathrm{HCO}^{+}$, we can only model the blue line wing, since the 3-2 red line wing is blended with the HCN 3-2 $v_{2}=1$ vibrational line. The blue line wings are also blended with $\mathrm{HOC}^{+}$lines at $-1000 \mathrm{~km} \mathrm{~s}^{-1}$, so to remove any contribution from this we only integrate between -820 and $-350 \mathrm{~km} \mathrm{~s}^{-1}$. The brightness temperature ratios for the blue wing of $\mathrm{HCO}^{+}$are 1:0.5:0 for $1-0: 2-1: 3-2$ (the $3-2$ line wing is not significantly detected; cf. Col. 7 in Table 3). If we assume the same cloud sizes as derived from the $\mathrm{HCN}$ observations, we find that the data are consistent with $X\left(\mathrm{HCO}^{+}\right) \lesssim 0.001 X(\mathrm{HCN})$ (see Fig. 6). However, considering the clumpy structure and chemical differentiation of the outflow discussed above, this ratio should vary significantly across the outflow. Assuming that the blue and red $\mathrm{HCO}^{+}$outflows are similar in mass, we reach outflow masses $M_{\text {dense }}=2.0 \times 10^{10} M_{\odot}$ for $X\left(\mathrm{HCO}^{+}\right)=10^{-9}$ and $M_{\text {dense }}=2.0 \times 10^{11} M_{\odot}$ for $X\left(\mathrm{HCO}^{+}\right) \stackrel{\odot}{=}$ $10^{-10}$. These masses are considerably higher than and inconsistent with those calculated for the total HCN outflow. They are also unrealistically high.

If we instead assume non-self-gravitating clouds for HCN and $\mathrm{HCO}^{+}$(which corresponds to $\Delta v \gg \sqrt{G M / R}$; in the lower panels of Fig. 6 we have assumed $\Delta v=10 \sqrt{G M / R}$ ), we reach lower outflow masses, in agreement with Aalto et al. (2015a). Compared to the self-gravitating clouds, the outflow masses calculated for both the $\mathrm{HCN}$ and $\mathrm{HCO}^{+}$components are approximately a factor of 5 lower when assuming $\Delta v=10 \sqrt{G M / R}$ but keeping the $\mathrm{HCN}$ and $\mathrm{HCO}^{+}$abundances unchanged. Thus, when assuming identical physical properties of the $\mathrm{HCN}$ and $\mathrm{HCO}^{+}$outflows and non-self-gravitating clouds, the $\mathrm{HCN}$ and $\mathrm{HCO}^{+}$models produce strongly contradictory outflow masses, 
just as when assuming self-gravitating clouds. The modelled $\mathrm{HCO}^{+}$outflow masses are also so high that the model assumptions must be incorrect (e.g. the temperature is too high), while those same assumptions appear to work for HCN. Consequently, the models support the previous conclusion of a chemical differentiation between $\mathrm{HCN}$ and $\mathrm{HCO}^{+}$in the molecular outflow.

\subsection{Variable continuum}

The high variability detected in the $3 \mathrm{~mm}, 1 \mathrm{~mm}$, and $1.5 \mathrm{~cm}$ $(20 \mathrm{GHz})$ continuum emission of Mrk 231 on timescales of a few years indicates that Mrk 231 is a blazar.

The time coverage of our $3 \mathrm{~mm}$ observations is unfortunately not good enough for a reliable modelling of the flaring event of Mrk 231. We can, however, discuss the consistency between our results and those at other frequencies (Aalto et al. 2012a, 2015a; Reynolds et al. 2013) in the frame of the standard jet model (e.g. Marscher 1980; Blandford \& Königl 1979).

The light curve at $20 \mathrm{GHz}$ reported in Reynolds et al. (2013) peaks in early March 2013 when the flux density roughly doubled, compared to its value in early January 2013. Our flux density at $3 \mathrm{~mm}$ in January 2013 is, however, already twice that reported by Aalto et al. (2012a). A similar result is found at $1 \mathrm{~mm}$, as already mentioned in Sect. 3: the flux density, compared to that in 2012, doubled in February 2013 (i.e. well before the peak intensity was reached at $20 \mathrm{GHz}$ in March 2013). The flux density at $\mathrm{mm}$ wavelengths thus seems to have varied by a factor of $\sim 2$ well before the peak intensity at $20 \mathrm{GHz}$ was reached. This is indicative of opacity effects in the jet (e.g. Blandford \& Königl 1979), which at lower frequencies would make the jet opaque at shorter distances to its base. Hence, flaring activity propagating downstream of the jet would be detectable earlier at higher frequencies and later at lower frequencies.

\section{Conclusions}

In this paper we present high-resolution observations of the outflow of the ULIRG Mrk 231. The galaxy has a very complex outflow structure, which is barely resolved in the PdBI observations. We have examined the chemical composition of this gas, and identify the following signs of chemical differentiation in the outflow:

1. The HCN line core emission of Mrk 231 is broader than the $\mathrm{HCO}^{+}$and $\mathrm{HNC}$ line core emission. This could represent $\mathrm{HCN}$ emission at forbidden velocities, but is more likely caused by optical-depth broadening of the HCN line.

2. The $\mathrm{HCN}, \mathrm{HCO}^{+}$, and $\mathrm{HNC}$ emission line wings all show features at different velocities, suggesting a clumpy, chemically differentiated outflow.

3. By radiative transfer modelling we show that while the $\mathrm{HCN}$ emission is consistent with a clumpy outflow, the same model properties applied to the $\mathrm{HCO}^{+}$emission generates unrealistically high outflow masses, which are also inconsistent with the HCN modelling results. This suggests that the $\mathrm{HCN}$ and $\mathrm{HCO}^{+}$emission originates in structures with different physical properties.

The chemical differentiation of $\mathrm{HCN}$ and $\mathrm{HCO}^{+}$can be explained by an enhancement of the $\mathrm{HCN} / \mathrm{HCO}^{+}$abundance ratio in shocked parts of the outflows (Mitchell \& Deveau 1983). At the highest velocities the $\mathrm{HCO}^{+}$emission traces pre-shock gas (possibly at a lower temperature than the $\mathrm{HCN}$ ). The HCN emission traces recently ejected gas interacting in a shock with the surrounding gas. The HNC appears at the lowest velocities, and traces the braked gas after the shock.
We also report that the $3 \mathrm{~mm}$ continuum flux of Mrk 231 was significantly enhanced by the radio flare event reported by Reynolds et al. (2013), indicating that this galaxy is a blazar.

Future observations using NOEMA could be used to achieve data with higher sensitivity and resolution, which is required to further investigate the nature of the complex outflow structure of this interesting object. This would improve our understanding of the origin of the outflows from ULIRGs. To better study the importance of shocked gas, such observations should not only target $\mathrm{HCN}$ and $\mathrm{HCO}^{+}$, but also shock tracers such as $\mathrm{SiO}, \mathrm{CH}_{3} \mathrm{OH}$, and $\mathrm{HNCO}$.

Acknowledgements. We thank the IRAM PdBI staff for excellent support. This research was supported by J.E.L.'s appointment to the NASA Postdoctoral Program at the NASA Goddard Space Flight Center, administered by Oak Ridge Associated Universities through a contract with NASA. S.A. thanks the Swedish National Science Council for grant support. We also thank the referee Chiara Feruglio for comments and suggestions which significantly contributed to improving the quality of the manuscript.

\section{References}

Aalto, S., Garcia-Burillo, S., Muller, S., et al. 2012a, A\&A, 537, A44 Aalto, S., Muller, S., Sakamoto, K., et al. 2012b, A\&A, 546, A68 Aalto, S., Garcia-Burillo, S., Muller, S., et al. 2015a, A\&A, 574, A85 Aalto, S., Martín, S., Costagliola, F., et al. 2015b, A\&A, 584, A42 Alatalo, K. 2015, ApJ, 801, L17

Alatalo, K., Blitz, L., Young, L. M., et al. 2011, ApJ, 735, 88 Blandford, R. D., \& Königl, A. 1979, ApJ, 232, 34

Bolatto, A. D., Warren, S. R., Leroy, A. K., et al. 2013, Nature, 499, 450

Bryant, P. M., \& Scoville, N. Z. 1996, ApJ, 457, 678

Carilli, C. L., Wrobel, J. M., \& Ulvestad, J. S. 1998, AJ, 115, 928

Cicone, C., Feruglio, C., Maiolino, R., et al. 2012, A\&A, 543, A99

Cicone, C., Maiolino, R., Sturm, E., et al. 2014, A\&A, 562, A21

Costagliola, F., Aalto, S., Rodriguez, M. I., et al. 2011, A\&A, 528, A30

Downes, D., \& Solomon, P. M. 1998, ApJ, 507, 615

Feruglio, C., Maiolino, R., Piconcelli, E., et al. 2010, A\&A, 518, L155

Feruglio, C., Fiore, F., Carniani, S., et al. 2015, A\&A, 583, A99

Fischer, J., Sturm, E., González-Alfonso, E., et al. 2010, A\&A, 518, L41

Gallagher, S. C., Brandt, W. N., Chartas, G., Garmire, G. P., \& Sambruna, R. M. 2002, ApJ, 569, 655

García-Burillo, S., Combes, F., Usero, A., et al. 2014, A\&A, 567, A125

Goldsmith, P. F. 1987, in Interstellar Processes, eds. D. J. Hollenbach, \& H. A.

Thronson, Jr., Astrophys. Space Sci. Libr., 134, 51

González-Alfonso, E., Fischer, J., Graciá-Carpio, J., et al. 2014, A\&A, 561, A27

Jiang, X., Wang, J., \& Gu, Q. 2011, MNRAS, 418, 1753

Lipari, S., Sanchez, S. F., Bergmann, M., et al. 2009, MNRAS, 392, 1295

Marscher, A. P. 1980, ApJ, 235, 386

Matsushita, S., Trung, D.-V., Boone, F., et al. 2015, ApJ, 799, 26

Mitchell, G. F., \& Deveau, T. J. 1983, ApJ, 266, 646

Müller, H. S. P., Thorwirth, S., Roth, D. A., \& Winnewisser, G. 2001, A\&A, 370, L49

Murray, N., Quataert, E., \& Thompson, T. A. 2005, ApJ, 618, 569

Nakai, N., Hayashi, M., Handa, T., et al. 1987, PASJ, 39, 685

Podio, L., Lefloch, B., Ceccarelli, C., Codella, C., \& Bachiller, R. 2014, A\&A, 565, A64

Reynolds, C., Punsly, B., O’Dea, C. P., \& Hurley-Walker, N. 2013, ApJ, 776, L21

Rupke, D. S. N., \& Veilleux, S. 2011, ApJ, 729, L27

Sakamoto, K., Ho, P. T. P., \& Peck, A. B. 2006, ApJ, 644, 862

Sakamoto, K., Aalto, S., Evans, A. S., Wiedner, M. C., \& Wilner, D. J. 2010 ApJ, 725, L228

Sturm, E., González-Alfonso, E., Veilleux, S., et al. 2011, ApJ, 733, L16

Tafalla, M., \& Hacar, A. 2013, A\&A, 552, L9

Tafalla, M., Santiago-García, J., Hacar, A., \& Bachiller, R. 2010, A\&A, 522, A91

Taylor, G. B., Silver, C. S., Ulvestad, J. S., \& Carilli, C. L. 1999, ApJ, 519, 185

Teng, S. H., Brandt, W. N., Harrison, F. A., et al. 2014, ApJ, 785, 19

Tsai, A.-L., Matsushita, S., Nakanishi, K., et al. 2009, PASJ, 61, 237

Usero, A., García-Burillo, S., Fuente, A., Martín-Pintado, J., \& Rodríguez-Fernández, N. J. 2004, A\&A, 419, 897

van der Tak, F. F. S., Black, J. H., Schöier, F. L., Jansen, D. J., \& van Dishoeck, E. F. 2007, A\&A, 468, 627

Walter, F., Weiss, A., \& Scoville, N. 2002, ApJ, 580, L21

Wang, M., Henkel, C., Chin, Y.-N., et al. 2004, A\&A, 422, 883 


\section{Appendix A: PV diagrams}

Here we show the $\mathrm{PV}$ diagrams for each individual $\mathrm{HCN}$ and $\mathrm{HCO}^{+}$line reported for the first time in this work. They were all obtained by a cut through the continuum peak, either in the north-south or east-west direction. All contours start at $2 \sigma$ and continue with $2 \sigma$ spacing (see Table 1 for rms levels).
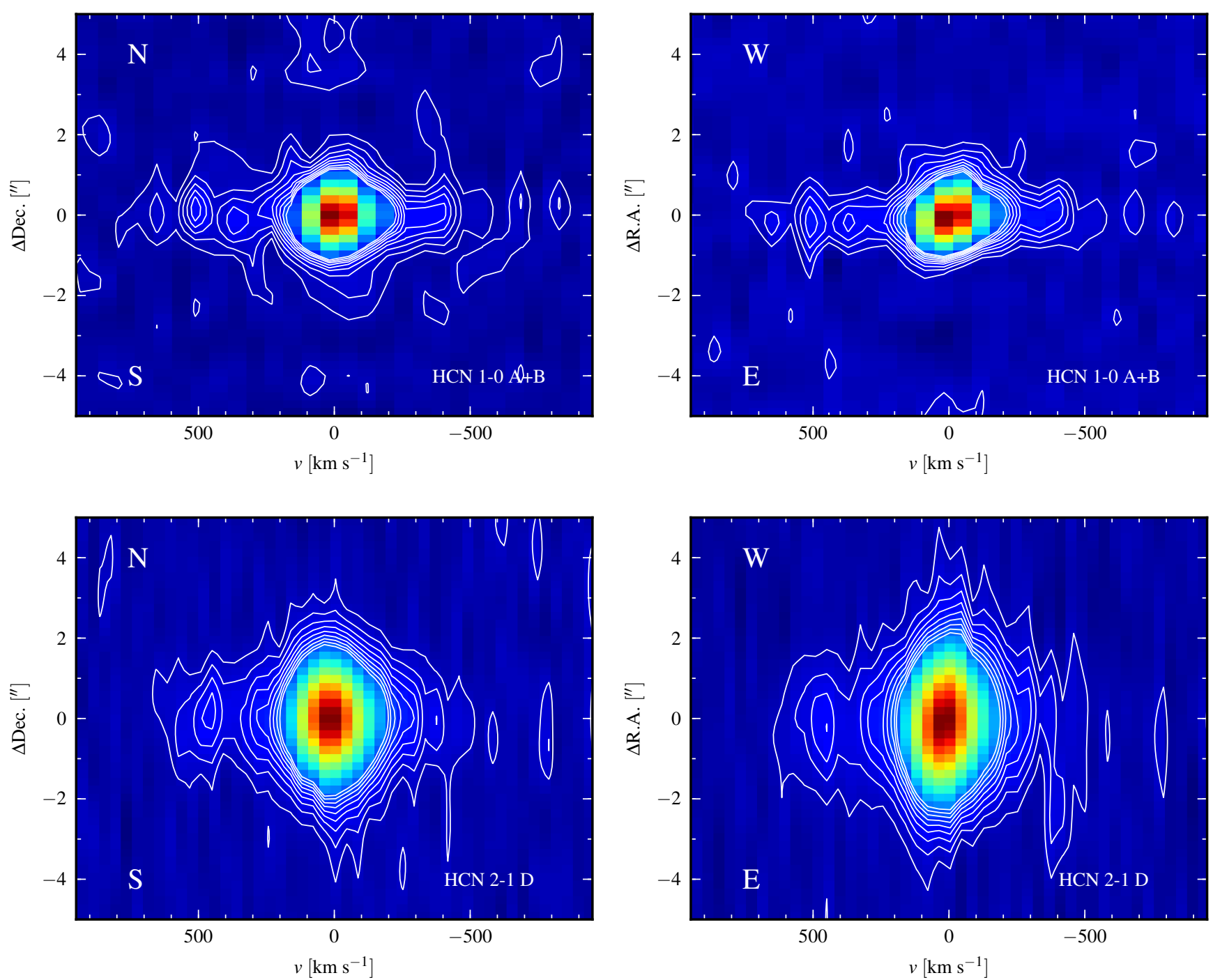

Fig. A.1. PV diagrams of individual $\mathrm{HCN}$ transitions (contours at $2 \sigma$ ). The HCN 1-0 PV diagram uses a combination of PdBI A-array and B-array observations. The HCN 2-1 PV diagram uses PdBI D-array observations. 

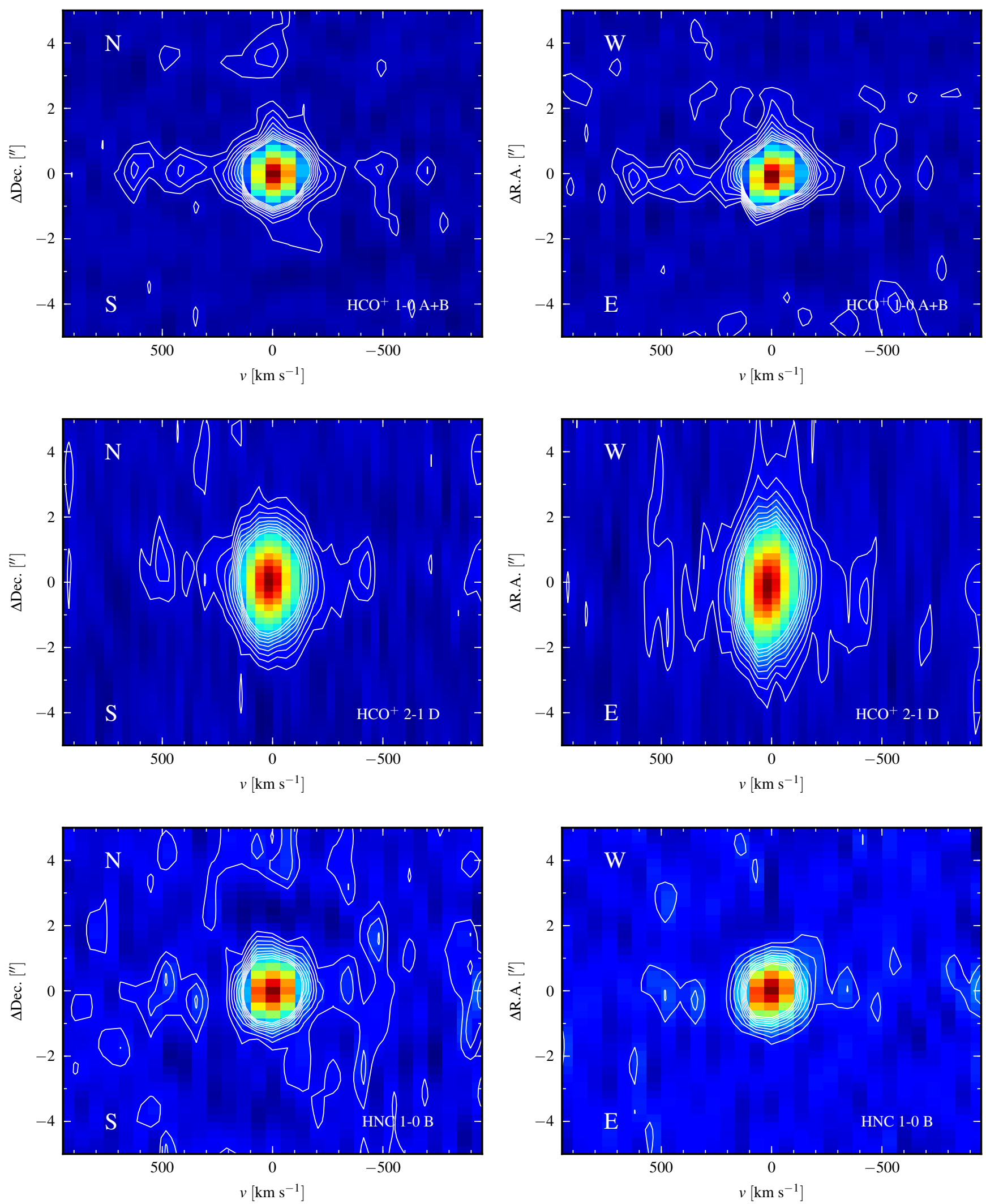

Fig. A.2. $\mathrm{PV}$ diagrams of individual $\mathrm{HCO}^{+}$and $\mathrm{HNC}$ transitions (contours at $2 \sigma$ ). The $\mathrm{HCO}^{+} 1-0 \mathrm{PV}$ diagram uses a combination of $\mathrm{PdBI} \mathrm{A}$-array and $\mathrm{B}$-array observations. The $\mathrm{HCO}^{+} 2-1 \mathrm{PV}$ diagram is made from PdBI D-array observations. The HNC $1-0$ PV diagram uses PdBI B-array observations. 